Maria Griemmert

\title{
Von Virenschleudern und Partygängern. Die mediale (Re-)Präsentation von Kindern und Jugendlichen in der Pandemie
}

Als Medizinhistorikerin am Institut für Geschichte, Theorie und Ethik der Medizin der HHU Düsseldorf beschäftige ich mich mit der Kindheitsgeschichte, und damit auch mit dem sich über die Zeit wandelnden gesellschaftlichen „Bild“vom Kind. Inwiefern die Corona-Pandemie Einfluss auf unser derzeitiges Kindesbild genommen hat und weiterhin nimmt, ist Teil meiner aktuellen Forschung.

Am 27. Januar 2020 wurde in Deutschland der erste Fall einer Erkrankung mit dem neuartigen Coronavirus bestätigt. Am 16. März 2020 begann der erste Lockdown, der die Lebenswelt von Kindern und Jugendlichen in hohem Maße veränderte: Schulen und Kindertagesstätten blieben für viele Wochen geschlossen, Kinderspielplätze wurden abgesperrt, Kinder- und Jugendhilfeeinrichtungen schlossen bzw. schalteten auf Notbetrieb und in öffentlichen Ämtern bestand ein Betretungsverbot für Kinder. Eltern waren angehalten, ihre Kinder nicht mit zum Einkaufen zu nehmen und möglichst nicht von den Großeltern betreuen zu lassen. Gerade Kinder, die altersbedingt noch keine Gänge allein bewältigen konnten, waren in dieser Zeit vollständig aus dem öffentlichen Raum ausgeschlossen (Bujans et al. 2021; Voigts 2020). In unzähligen Fenstern sah man während dieser Tage als Dokumentation der Selbstbeschränkung die bunten Regenbogen-Ausmalbilder mit dem Motto Wir bleiben zu Hause.

Wie und mit welchen Schwerpunkten wurden in diesen außergewöhnlichen Monaten familien- und jugendrelevante Themen in Deutschland medial adressiert, welche Narrative wurden bedient und welche Aspekte waren nur wenig präsent? Der nachfolgende Beitrag untersucht, welche Konjunkturen sich in der Berichterstattung zu Kindern und Jugendlichen am Beginn der Pandemie im deutschsprachigen Raum abzeichneten und welche(s) Bild(er) von Kindern und Jugendlichen zu Beginn der Corona-Pandemie in den Medien transportiert wurde. ${ }^{1}$

1 Untersucht wurde v. a. die Repräsentation kinder- und jugendrelevanter Themen in journalistisch erstellten online-Zeitungen und -News- sowie TV-Kanälen (vgl. Fußnote 2). Social-mediaBeiträge, Kommentare in Meinungsforen usw. wurden nicht berücksichtigt. 


\section{Corona, Kinder und Schulen: Die Fieberkurve der Berichterstattung}

In der Fieberkurve der Berichterstattung, die unangefochten vom Begriff Corona dominiert wurde, zeigten sich im Jahresverlauf 2020 sowohl deutliche Spitzen als auch Berichts-Täler (Abb. 1)². Diese korrespondierten mit einer Vielzahl von Faktoren, wie z. B. mit akuten Maßnahmen zum Infektionsschutz, steigenden Inzidenzwerten, Veröffentlichungen neuer Studien, Ferienzeiten etc. In Bezug auf kinder- und jugendrelevante Themen erfuhr im gleichen Zeitraum der Begriff Schulen im quantitativen Vergleich die stärkste Berichterstattung mit den höchsten Nennungs-Frequenzen, auch Kinder war vergleichsweise stark vertreten. ${ }^{3}$ Das Ringen um Schulschließungen oder (Wieder-)Öffnungen war in 2020 der große kinderund jugendspezifische Themenkomplex. Diese wurden medial zunächst vor allem unter praktischen Gesichtspunkten diskutiert: Stellen sie Eltern vor ein Betreuungsproblem? Funktionieren Distanzlernen, Home-Schooling und Lernplattformen? Kann das Abitur stattfinden und was ist das Corona-Abi wert? (vgl. Voigts 2020).

Die Etikettierung als Treiber der Pandemie wurde in Bezug auf Schulen ab Frühsommer 2020 in die öffentliche Debatte eingebracht - ursprünglich in negierender Argumentation, dass Schulen und Kindertagesstätten eben keine solchen seien und darum geöffnet werden bzw. bleiben sollten. Seitdem tobt ein Streit um die Deutungshoheit darüber, ob diese Institutionen - und die sie besuchenden Kinder und Jugendlichen - nun als solche Infektionstreiber gelten müssen, oder

\footnotetext{
2 Erstellt wurden die Charts mit dem „cOWID plus Viewer“ des Leibnitz Instituts für die deutsche Sprache, mit dem sich das sich wandelnde Vokabular deutschsprachiger Online-Pressemeldungen seit Beginn des Jahres 2020 explorieren lässt. In die Auswertung einbezogene Online-Zeitungen mit Print-Pendant: Focus Online, Frankfurter Allgemeine Zeitung / FAZ.net, Frankfurter Rundschau / fr.de, Süddeutsche Zeitung / SZon, Neue Zürcher Zeitung - Schweiz / NZZ.ch, Spiegel Online / SPon, Der Standard - Österreich / derstandard.at, tageszeitung, taz / taz.de, Die Welt/ Welt.de, Die Zeit/ ZON; online-Angebote ohne Print-Pendant: web.de, t-online.de, heise.de. Da es sich um eine Ressource zur deutschen Sprache und nicht Deutschlands handelt, wurden in die Auswertung der österreichische Standard und die Schweizer NZZ einbezogen. Quelle: https:// www.owid.de/plus/cowidplusviewer2020. Zugriff am 28. September 2021.

3 Die hier nicht dargestellte Kurve für den Begriff Kita folgt insgesamt dem Verlauf der Schulen auf etwas niedrigerem Häufigkeits-Niveau; die Nennung des Begriffs Jugendliche wäre weit unterhalb der Kinder-Kurve angesiedelt. Andere einschlägige Begriffe des Jahres (z. B. Home-Office, Impfung) zeigten sich demgegenüber im quantitativen Vergleich 2020 deutlich abgeschlagen mit einem Bruchteil der Nennungsfrequenzen von Schule/Kinder (owid.de 2020; siehe auch Fußnote 2).
} 
Gleitender Mittelwert mit Fenstergröße von 6 Tag(en)

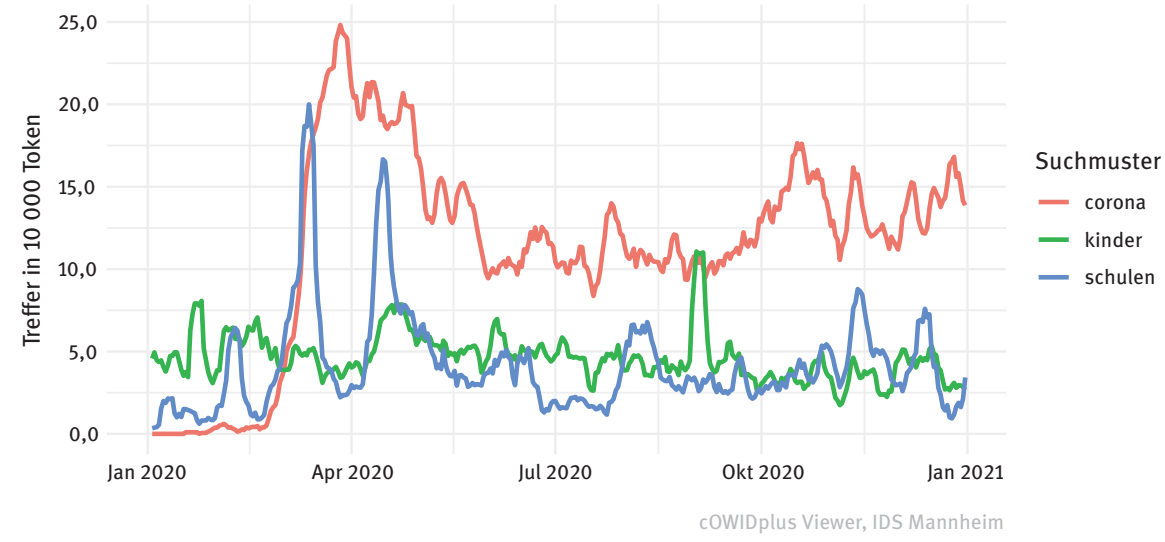

Abb. 1: Fieberkurven der Berichterstattung. Nennungshäufigkeit 2020 der Begriffe Corona, Kinder und Schulen in Pressemeldungen aus 13 deutschsprachigen Online-Medien (owid.de)

gerade nicht. Dieser Diskurs flammte 2020 immer dann neu auf, wenn neue Daten erschienen, aufgrund steigender Infektionszahlen politische Entscheidungen zu neuen Maßnahmen des Infektionsschutzes anstanden und/oder Ferienzeiten endeten. ${ }^{4}$

Insgesamt zeigte sich in 2020 eine starke Präsenz der Berichterstattung über Kinder hinsichtlich einer möglichen spezifischen Gefahr, die von ihnen als stille, selbst kaum erkrankende Virusüberträger für ihre Umwelt ausgehen könnte. In diesem Kontext ist auch der plakative und despektierliche, hier titelgebende Begriff des Kindes als Virenschleuder einzuordnen (vgl. Voigts 2020). ${ }^{5}$ Wie kam es, dass Kinder - die mittlerweile in Bezug auf die Verbreitung von COVID-19 von diesem Vorwurf rehabilitiert wurden - im ersten Jahr der Pandemie so stark in den Fokus der medialen Aufmerksamkeit rückten?

4 Die diskursiven Scharmützel um das Label Infektionstreiber hat Adler in einer Diskursanalyse nachvollzogen (Adler 2021).

5 In der journalistischen Berichterstattung ist der drastische Begriff Virenschleuder vor allem in seiner Negation oder in Anführungszeichen zu finden. Exempl.: Coronavirus: Sind Kinder „Virenschleudern“? (Durach, 14.6.2020, merkur.de); Weil wir nicht wissen, ob Kinder „Virenschleudern“ sind, sollten Schulen öffnen (Straubhaar, 11.6.2020, Welt.de); Kinder sind keine Virenschleudern (Bahnsen, 28.4.2021, ZON). Er hat aber vor allem auch in Meinungsforen und Kommentarspalten Eingang gefunden. 


\section{Kinder in der (in-)offiziellen Risikokommunikation um COVID-19}

In einer Krise wie der Corona-Pandemie, die im Frühjahr 2020 ihren Ausgang nahm, nimmt die Risikokommunikation im Aufklärungsprozess um Gefahren und richtige Verhaltensweisen eine wichtige Rolle ein. Laut Weltgesundheitsorganisation beinhaltet sie den „Austausch von Informationen, Empfehlungen und Meinungen zwischen Experten und der Bevölkerung angesichts von Bedrohungen für ihre Gesundheit und/oder ihr wirtschaftliches oder soziales Wohlergehen“ (WHO Definition, zitiert nach Loss et al. 2021).

Das vom Bundesministerium des Innern Mitte März 2020 in Auftrag gegebene Strategiepapier Wie wir COVID-19 unter Kontrolle bekommen (BMI 2020) stellte eine Art Leitfaden für die mediale Ansprache pandemiepolitischer Themen dar. ${ }^{6}$ Kinder werden in diesem Dokument in Kapitel 4. Schlussfolgerungen für Maßnahmen und offene Kommunikation als gesondert anzusprechende Gruppe adressiert:

4 a. Worst case verdeutlichen!

[...] Um die gewünschte Schockwirkung zu erzielen, müssen die konkreten Auswirkungen einer Durchseuchung auf die menschliche Gesellschaft verdeutlicht werden: [...]

2) „Kinder werden kaum unter der Epidemie leiden“: Falsch. Kinder werden sich leicht anstecken, selbst bei Ausgangsbeschränkungen, z. B. bei den Nachbarskindern. Wenn sie dann ihre Eltern anstecken und einer davon qualvoll zu Hause stirbt und sie das Gefühl haben, Schuld daran zu sein, weil sie z. B. vergessen haben, sich nach dem Spielen die Hände zu waschen, ist es das Schrecklichste, was ein Kind je erleben kann. [...] (BMI 2020, 13)

Die ausdrückliche Kopplung von Ansteckungen durch soziale Interaktion mit dem Begriff der Schuld bürdet Kindern und Jugendlichen hier rhetorisch eine schwere Verantwortung für das (Über-)Leben ihrer Nächsten auf. Aus einer klassischerweise als zu schützend angesehenen Bevölkerungsgruppe werden Kinder und Jugendliche hier durch eine (Über-)Betonung ihrer „als gefährlich wahrgenommenen Körperlichkeit“ (Alberth et al. 2021, 1) zu einer, vor der explizit die eigenen Eltern und Großeltern, aber auch die Gesellschaft geschützt werden müssten. Gleichzeitig rücken die eigenen Belange und Bedürfnisse von Kindern und Jugendlichen in den Hintergrund.

6 Am 27. März 2020 berichteten WDR, NDR und Süddeutsche Zeitung (Richter, Mascolo und Charisius, SZ, 27. März 2020); in den darauffolgenden Tagen weitere Medien (z. B. Alexander, Bewarder und Breyton 2020, Die Welt, 28. März 2020; Rosenfelder, Die Welt, 20.4.2020) über das Papier, das seit Ende April 2020 auf der Internetseite des Bundesministeriums des Innern online abrufbar ist (BMI 2020). 


\section{Jugendliche Partygänger: „Moral panics“ 2020}

Polizei beendet „Corona Partys“: Söder mahnt und droht. (dpa/ SZ 17.3.2020)

Was passiert, wenn die Corona-Party weitergeht? Junge Menschen sind die Hauptträger des Coronavirus, das zeigen aktuelle Zahlen. Doch ausgerechnet sie tun sich schwer damit, ihr Verhalten der Krise anzupassen. (Baum und Fischer, ZEIT Campus 17.3.2020)

Jugendliche husten Senioren an und rufen „Corona“ - nicht nur die Polizei ist entsetzt. (Mannheim24.de, 25.3.2020)

Im Frühjahr 2020 erschienen binnen weniger Wochen zahllose Medienberichte zu mutmaßlichen jugendlichen Verstößen gegen das Infektionsschutzgesetz. Sogenannte Corona-Partys feiernde Jugendliche standen hier im Fokus der Berichterstattung. Getrieben von missbilligenden Äußerungen verschiedener politischer Akteure wurde im Frühjahr 2020 durch die rein negative Präsenz von Jugendlichen in den Medien das Bild einer verantwortungslosen, egoistischen Generation entworfen, der es ohne Rücksicht auf Verluste vordringlich um ihren eigenen Spaß ginge (vgl. Voigts 2020; Gravelmann 2020).

Unter den Vorzeichen des beklagten jugendlichen Hedonismus wurde teils eine Spaltungstendenz des intergenerationellen Zusammenhaltes und ein dräuender Generationenkonflikt konstatiert. Hinter dem unverantwortlichen Verhalten der Jugend stecke demnach ein

gar unbarmherziges Kalkül: „Wir lassen uns doch nicht von denen, [...] der Risikogruppe, den Alten, den Spaß verderben. Weniger Alte, bedeutet das nicht auch mehr Rente für mich?“ [...] Auch der Generationenkonflikt köchelt also im Hintergrund des Virusgeschehens. (Pauls 2020, zitiert nach Okunew und Theinert 2020)

Verschiedene Untersuchungen haben sich zwischenzeitlich der Frage angenommen, inwiefern dieses medial sehr aufgeregt diskutierte und skandalisierte jugendliche Verhalten wirklich systematischen Charakter hatte. Jugendstudien der jüngsten Zeit, wie z. B. der TUI-Stiftung (TUI 2020 und 2021) oder die Sonderauswertung der Studie Junge Deutsche (Schnetzer und Hurrelmann 2021) kommen übereinstimmend zu dem Schluss, dass die Jugendlichen in überwältigender Mehrheit die geforderten Distanz- und Hygiene-Vorgaben erfüllten. Gleichzeitig hielten die medial laut beklagten Ereignisse in einigen Fällen der näheren journalistischen Tatsachenüberprüfung nicht stand (vgl. Petter und Eberle 2021).

Worauf beruhte dann die massive Welle negativer Berichterstattung im Frühling 2020? Es kursieren verschiedenste Deutungsangebote für das Phänomen der 
harschen, zwischenzeitlich als überzogen entlarvten Jugendkritik, von denen einige im Folgenden in Kürze dargestellt werden:

- Die vorgebrachte Jugendkritik wird als uraltes Phänomen gedeutet, das seit (mindestens) der Antike zu allen Zeiten und in allen Gesellschaften zu finden sei (z. B. Warkus 2020). ${ }^{7}$

- Das Narrativ einer generellen jugendlichen Bereitschaft, sich aus Hedonismus gegen gesamtgesellschaftliche Interessen zu stellen, diene (für die zuschreibende ältere Generation) der Wiederherstellung der moralischen Ordnung: Nachdem 2019 die Fridays-for-Future-Bewegung den Finger in die Wunde legte und der älteren Generation implizit wie explizit vorwarf, nicht verantwortungsvoll mit der Zukunft ihrer Kinder und Enkelkinder umzugehen, werde nun durch das kategorisch als egoistisch abgeurteilte Verhalten der Jungen in der Pandemie die moralische Ordnung wiederhergestellt. ${ }^{8}$

- Die Autoren Okunew und Theinert sehen sich dagegen durch Merkmale der Berichterstattung an frühere mediale Skandalisierungen jugendlicher Normabweichungen erinnert. Im soziologischen Kontext wurde in den 1970er Jahren der Begriff der moral panic geprägt. ${ }^{9}$ Dieser beschreibt ein Phänomen, das in der deutschen Geschichte z. B. für die Berichterstattung über die sog. Halbstarken präsent war: Merkmal dieses Phänomens ist die überproportionale mediale Skandalisierung von jugendlicher Normabweichung, deren Berichtsfülle und -charakter in keinem adäquaten Verhältnis zum tatsächlichen Vorfall steht. Gedeutet wird dieser Mechanismus als eine Art Katalysator gesellschaftlicher Ängste vor Veränderung. Der immense öffentlich-moralische Druck durch das Empörungspotential führt zu einer Stigmatisierung abweichenden Verhaltens und damit zu verstärkter sozialer Kontrolle (Okunew und Theinert 2020). Folgt man dieser Analyse, könnten Jugendliche hier also als Sündenböcke und Projektionsfläche für gesellschaftliche Ängste bezüglich der (Un-)Kontrollierbarkeit der Pandemie gedient haben (ähnlich der

\footnotetext{
7 Ein in diesem Zusammenhang mit diesem Argument häufig anzutreffender Verweis auf die Klage des Sokrates (469-399 v. Chr.) vor mehr als 2000 Jahren über eine faule, respektlose Jugend ohne Manieren hat sich zwischenzeitlich jedoch als Zitat-Erfindung des 20. Jahrhunderts herausgestellt (Warkus 2020; O’Toole 2010).

8 Der sich hier andeutende Diskurs um Generationengerechtigkeit erhielt in den folgenden Monaten eine weitere Wendung: Die vergleichsweise großen und (zumindest teilweise) fremdnützigen Opfer der jungen Generation zum Schutz der Älteren wurden anerkannt und darauf basierend Forderungen nach einer intergenerationellen Solidarität in Bezug auf z. B. Klimaschutzziele formuliert (vgl. ZDF 20.11.2020; Spittler 2020).

9 Der Soziologe Stanley Cohen untersuchte den medial aufgebauschten und skandalisierten Konflikt zweier rivalisierender Gruppen von devianten Jugendlichen im England der 1960er Jahre (Cohen 1972).
} 
kurzzeitigen medialen Skandalisierung schlittenfahrender Familien wenige Monate später).

Aus welcher Quelle sich die mediale Empörungswelle genuin auch speiste, die sich ihrerseits im Nachgang den Vorwurf einer ,von Ressentiments geleiteten Verleumdung der Jugendlichen und eine Verzerrung der Realität“ (Klundt 2021, 100) einhandelte: Sie kann als Symptom einer Verhärtung und Verunsachlichung der Debattenkultur in einer gesellschaftlichen Krisensituation begriffen werden.

Zeigte sich in der Empörung(sbereitschaft) rund um deviantes Jugendverhalten die überproportional hohe mediale Präsenz eines - offenbar zumindest weitgehend herbeigeschriebenen - Phänomens, so gibt es andere kinder- und jugendrelevante Themen, die demgegenüber im gleichen Zeitraum eher unterrepräsentiert waren.

\section{4 (Strukturelle) Gefährdungslagen 2020: Dunkelfeld der Berichterstattung}

Gerade zu Beginn des ersten Lockdowns wurden Maßnahmen zur Pandemiebekämpfung, die Gefahren für das Kindeswohl mit sich brachten, medial nur wenig problematisiert. ${ }^{10}$

Der unvermittelte Entzug von Hilfe- bzw. Kontrollmechanismen, die darauf abzielen, vulnerable Kinder und Jugendliche aus besonders belasteten Familien zu schützen, hatte Folgen für das Kindeswohl in einer Reihe von Fällen (Holz und Richter-Kornweitz 2020). Gleichzeitig aber gingen Meldungen von Kindeswohlgefährdungen in diesem Zeitraum deutlich zurück (vgl. PM Statistisches Bundesamt vom 17.9.2021). Dieser hier entstehende blinde Fleck der medialen Agenda lässt sich u. a. mit der verstärkten Berichterstattung um die Bedrohungslage bezüglich des COVID-19 Virus erklären. Hier geschah offenbar eine Art Agenda Cutting, das

10 Einige Maßnahmen zum Infektionsschutz hatten einen direkten negativen Einfluss, gerade auf die Gruppe der besonders vulnerablen Kinder und Jugendlichen. Hierzu zählten 2020 z. B. die wochenlange und teilweise ersatzlose Schließung der Tafeln, deren Angebote nach eigenen Angaben ca. 500.000 Kinder regelmäßig bedürfen, das Aussetzen von Angeboten und Hilfestellungen der Jugendhilfe, das Aussetzen der Vorsorge- und Schuleingangsuntersuchungen, die als Anhaltspunkte für Entwicklungsstörungen und Misshandlungen dienen können, die längerfristige Unterbrechung von pädagogischen und therapeutischen Hilfen für Kinder mit besonderem Förderbedarf und ihre Familien, das Aussetzen von Besuchen von Familienhebammen, strikte Besuchsregeln in Kliniken, durch die Eltern und Kinder zum Teil für Wochen getrennt wurden etc. (vgl. Bujard et al. 2021). 
andere Themenfelder längerfristig überlagerte bzw. in den Hintergrund treten ließ (Rieg 2020).

Das ohnehin große Dunkelfeld innerfamiliärer Gewalt entwickelte sich in der Krise, insbesondere durch die kontaktbeschränkenden Maßnahmen zum Infektionsschutz, zur völligen Blackbox, deren unmittelbare Bedrohlichkeit für Betroffene medial und politisch nur wenig Resonanz erfuhr. ${ }^{11}$ Die zwischenzeitlich erhobenen Zahlen belegen, dass 2020 in Hinsicht auf die Gesamtzahl gemeldeter Kindeswohlgefährdungen - trotz der Meldelücke während der Kontaktbeschränkungen im Frühjahr - einen traurigen Rekord aufstellt (PM Statistisches Bundesamt vom 17.9.2021).

Exemplarisch soll hier kurz auf den Fall Fabio verwiesen werden, der vergleichsweise nur wenig mediale Resonanz erfuhr: Laut Medienberichten war der 5-jährige Junge im April 2020 in Mönchengladbach durch Gewalteinwirkung des Lebensgefährten seiner Mutter zu Tode gekommen. Umstände und Vorgeschichte der Tat enthalten Elemente, die in der Vergangenheit seit dem Fall Kevin 2006 regelmäßig zur medialen Skandalisierung ähnlicher Fälle führte, in denen „Vernachlässigung als Leittopos eines versagenden Kinderschutzes“ aufgegriffen wurde (Görgen et al. 2013, 224).

Im Fall Fabio - wie auch in anderen ähnlich gelagerten Kinderschutzfällen kam es unter dem Eindruck der Pandemie 2020 jedoch nicht $\mathrm{zu}$ hohen medialen Diskurskonjunkturen, die im Nachgang ein politisches Agenda Setting oder eine gesellschaftliche Debatte ausgelöst hätten. ${ }^{12}$ Die auffällige Ruhe, die in diesem Zeitraum in Bezug auf die mediale Aufmerksamkeit für die Belange von Kindern und Jugendlichen und ihren Familien herrschte, hatte sich also als trügerisch erwiesen.

11 Anders stellt es sich bei den großen Missbrauchsskandalen der jüngsten Vergangenheit in Lügde (Winter 2018/19), Bergisch Gladbach (Herbst 2020), Münster (Juni 2021) dar, deren gesellschaftliches Empörungspotential durch die Dokumentation der Taten in Form der Erstellung kinderpornographischen Materials gesteigert wurde und in einem Gesetzgebungsverfahren mit der Zielsetzung eines besseren Schutzes von Kindern vor sexualisierter Gewalt mündete (Gesetz zur Bekämpfung sexualisierter Gewalt gegen Kinder, Bundesgesetzblatt Jahrgang 2021 Teil I Nr. 33; 22.6.2021, online abrufbar unter: https://www.bmjv.de/SharedDocs/ Gesetzgebungsverfahren/Dokumente/Bgbl_Bekaempfung_sexualisierte_Gewalt_gegen_Kinder. pdf. Zugriff am 28. September 2021).

12 Die mediale Berichterstattung um den Tod des Jungen beschränkte sich im April 2020 auf wenige, vorwiegend regionale Meldungen (exemplarisch für Art und Umfang der Berichterstattung, z. B.: Gruhn und Peters / RP 22.4.2020; Wojtuschak / Bild.de Regional Düsseldorf, 22.4.2020; RTL news.de, 24.4.2020). Der Gerichtsprozess im Winter 2020/21 erfuhr etwas mehr Aufmerksamkeit, die kritische Hinterfragung nach einer gesellschaftlich-politischen Verantwortung blieb jedoch die Ausnahme (vgl. Hell und Kampf / SZon, 3.12.2020; Sartory WDR 5 Westblick, 15.1.2021; Helmig / Brisant, NDR, 3.3.2021; Büscher und Henrichs / Welt.de, 11.10.2021). 


\section{Fazit und Ausblick}

Kinder und Jugendliche dienten in der ersten Phase der Corona-Pandemie aufgrund ihrer früh erkannten geringen gesundheitlichen Gefährdungseinschätzung durch das neue Virus im deutschen medialen Diskurs verschiedentlich als Mittel zum Zweck pandemiepolitischer Maßnahmen und der zugehörigen Risikokommunikation.

Es zeigte sich, dass Kinder und Jugendliche zumeist als Mitglieder der - aus epidemiologischem Blickwinkel - nicht unproblematischen Institutionen Kita und v. a. Schule begriffen und adressiert wurden. Die (Über-)Betonung einer Verantwortlichkeit von Kindern und Jugendlichen für die Gesundheit der Älteren gegenüber ihrem eigenen Anspruch auf gesellschaftlichen Schutz und Teilhabe ist seit Pandemiebeginn als kontinuierlicher Subtext in der medialen Berichterstattung zu finden. Seinen Ausgang nahm dieses nur schwer zu revidierende Narrativ möglicherweise in der unmittelbar auf eine Gefährlichkeit der Kinder ausgerichteten Risikokommunikationsstrategie der Bundesregierung. Zu untersuchen wird sein, ob und in welchem Umfang diese Strategie in den Medien aufgegriffen wurde und, falls ja, welchen Einfluss sie auf das medial transportierte Bild des Kindes in der Pandemie und auch die getroffenen politischen Entscheidungen hatte und vielleicht noch immer hat.

Die Medienberichterstattung des nunmehr zweiten pandemischen Jahres 2021 im Hinblick auf Kinder und Jugendliche war seit Beendigung des zweiten Lockdowns zum einen geprägt von der ernüchternden Erkenntnis der vielfältig massiven negativen Auswirkungen der politischen Maßnahmen zum Infektionsschutz speziell auf diese Gruppe: In Hinsicht auf Faktoren, wie beispielsweise eine Verschlechterung der psychosozialen Gesundheit, den Anstieg von Kindeswohlgefährdungen, die Verringerung von Lebenschancen durch Bildungsdefizite und gebrochene Bildungsbiographien etc. gelten Kinder und Jugendliche inzwischen offiziell als Verlierer der pandemiepolitischen Maßnahmen in Deutschland. ${ }^{13}$

Neben diesen eher düsteren Rückmeldungen aus der Wissenschaft, von Ärzteverbänden und Kinderschutzorganisationen bestimmt im Herbst 2021, zum Zeitpunkt der Drucklegung dieses Bandes, jedoch vor allem eine Debatte um die Impfung der Unter-18-Jährigen zur Erreichung der erwünschten Impfquote den medial-politischen Diskurs über Kinder und Jugendliche. Es scheint, dass sie 2021 noch nicht aus der gesellschaftlichen Verantwortung entlassen werden.

13 Den Forschungsstand bis Mitte 2021 zu den „Belastungen von Kindern und Jugendlichen in der Corona-Pandemie“ fassen Bujard et al. für das Bundesinstitut für Bevölkerungsforschung zusammen (Bujard et al. 2021). 


\section{Literatur}

Adler D (2021) „Infektionstreiber“ im Corona-Diskurs: Der diskursive Kampf um die Lasten der Pandemiebekämpfung In: DiscourseNet. Collaborative Working Paper Series (4). http://dx. doi.org/10.17613/2d40-4h45.

Alberth L, Bollig S, Schindler L (2020) Materialitäten der Kindheit: Analytische Sichtachsen auf Körper, Dinge und Räume. In: Bollig S, Alberth L, Schindler L (Hg) Materialitäten der Kindheit. Körper - Dinge - Räume. Wiesbaden: Springer VS (Kinder, Kindheiten und Kindheitsforschung), Band 22: 1-14.

Alexander R, Bewarder M, Breyton R (2020, 28. März) Geht es um den „Exit“ - oder gar eine Verschärfung? Die Welt, S. 4. / Welt online https://www.welt.de/politik/deutschland/ plus206852527/Corona-Geht-es-um-den-Exit-oder-gar-eine-Verschaerfung.html. Zugriff am 9. August 2021.

Bahnsen U (2021, 28. April) Kinder sind keine Virenschleudern. DIE ZEIT 18/2021 / ZEIT ONLINE https://www.zeit.de/2021/18/coronavirus-kinder-infektion-schule-israel-forschung. Zugriff am 9. August 2021.

Baum C, Fischer L (2020, 17. März) Was passiert, wenn die Corona Party weitergeht? Junge Menschen sind die Hauptträger des Coronavirus, das zeigen aktuelle Zahlen. Doch ausgerechnet sie tun sich schwer damit, ihr Verhalten der Krise anzupassen. ZEIT Campus online, https://www.zeit.de/campus/2020-03/coronavirus-junge-menschen-ausbreitungverhaltensaenderung. Zugriff am 9. August 2021.

Bernhard A (2021) Lockdown und soziale Distanzierung - Anmerkungen zu einem (unfreiwilligen?) gesellschaftspädagogischen Experiment und seinen Folgen. In: Lutz R, Steinhaußen J, Kniffki J (Hg) Corona, Gesellschaft und Soziale Arbeit: Neue Perspektiven und Pfade. Weinheim: Beltz Verlagsgruppe, 49-60.

BMI (2020) Wie wir COVID-19 unter Kontrolle bekommen. Strategiepapier des Bundesministerium des Innern / März 2020. https://www.bmi.bund.de/SharedDocs/downloads/DE/ veroeffentlichungen/2020/corona/szenarienpapier-covid19.html. Zugriff am 9. August 2021.

Büscher W, Hinrichs P (2021, 11. Oktober) Das lange Sterben des kleinen Fabio. Wenn der Staat versagt, Teil 3. Welt.de, https://www.welt.de/politik/plus234258332/Staatsversagen-inMoenchengladbach-Das-lange-Sterben-des-kleinen-Fabio.html. Zugriff am 15. Oktober 2021.

Bujard M, von den Driesch E, Ruckdeschel K, Laß I, Thönnissen C, Schumann A, Schneider N. F. (2021) Belastungen von Kindern, Jugendlichen und Eltern in der Corona-Pandemie. Bundesinstitut für Bevölkerungsforschung (Hg) BIB. Bevölkerungs. Studien. https://www.bib. bund.de/Publikation/2021/pdf/Belastungen-von-Kindern-Jugendlichen-und-Eltern-inder-Corona-Pandemie.pdf. Zugriff am 9. August 2021.

Charisius H, Mascolo G, Richter N. (2020, 27. März) Innenministerium dringt auf massive Ausweitung von Corona-Tests. Süddeutsche Zeitung online, https://www.sueddeutsche.de/ politik/coronavirus-tests-strategie-1.4858950. Zugriff am 9. August 2021.

Cohen S (1972) Folk Devils and Moral Panics. St Albans: Paladin.

DPA (2020, 17.März) Polizei beendet „Corona Partys“: Söder mahnt und droht. Süddeutsche Zeitung online, https://www.sueddeutsche.de/gesundheit/gesundheit-nuernbergpolizei-beendet-corona-partys-soeder-mahnt-und-droht-dpa.urn-newsml-dpa-com20090101-200317-99-362067. Zugriff am 9. August 2021. 
Durach F (2020, 14. Juni) Coronavirus: Sind Kinder „Virenschleudern“? Neue Studien zeigen eine klare Tendenz. Merkur.de, https://www.merkur.de/welt/coronavirus-kinder-schulenansteckung-virologe-sars-cov-2-studien-kitas-infektion-zr-13795106.html. Zugriff am 9. August 2021.

Görgen A, Griemmert M, Fangerau H (2013) Kindheit und Trauma: Medikalisierung und Skandalisierung im Umgang mit der Gewalt an Kindern. Trauma \& Gewalt 7(3): 218-229.

Gravelmann R (2020) Jugend in Coronazeiten. Diskurse in Gesellschaft und Wissenschaft. In: Dialog Erziehungshilfe 04/2020. https://www.jugendhilfeportal.de/forschung/ jugendforschung/artikel/jugend-in-coronazeiten-diskurse-in-gesellschaft-undwissenschaft. Zugriff am 9. August 2021.

Gruhn A, Peters G (2020, 22. April) 23-Jähriger soll Fünfjährigen zu Tode geprügelt haben. RP online, https://rp-online.de/nrw/staedte/moenchengladbach/moenchengladbachfuenfjaehriger-junge-tot-mann-23-soll-ihn-totgepruegelt-haben_aid-50176199. Zugriff am 9. August 2021.

Hell A, Kampf L (2020, 3. Dezember) Fall Fabio: Gefährdete Kinder, die wegen Corona niemand mehr sieht. / Fall Fabio: Jugendamt sieht keine Fehler bei sich. WDR online, https://www1. wdr.de/nachrichten/gefaehrdete-kinder-fabio-corona-100.html. Zugriff am 9. August 2021.

Helmig S (Redaktion, 2021, 3. März) „Urteil Mönchengladbach: 5-Jähriger zu Tode geprügelt“ Brisant / Das Erste 12:00-12:25 Uhr, Beitrag in der Mediathek nicht mehr verfügbar.

Holz G, Richter-Kornweitz A (2020) Corona-Chronik - Gruppenbild ohne (arme) Kinder. Eine Streitschrift. Institut für Sozialarbeit und Sozialpädagogik e. V. (ISS). https://www.iss-ffm. de/fileadmin/assets/themenbereiche/downloads/Corona-Chronik_Streitschrift_final.pdf. Zugriff am 9. August 2021.

Klundt M (2021) Kinder, Kinderrechte und Kinderschutz im Corona-Kapitalismus. In: Lutz R, Steinhaußen J, Kniffki J (Hg) Corona, Gesellschaft und Soziale Arbeit: Neue Perspektiven und Pfade. Weinheim: Beltz Verlagsgruppe, 89-104.

Loss J, Boklage E, Jordan S, Jenny MA, Weishaar H, El Bcheraoui C (2021) Risikokommunikation bei der Eindämmung der COVID-19-Pandemie: Herausforderungen und Erfolg versprechende Ansätze. Bundesgesundheitsblatt Gesundheitsforschung, Gesundheitsschutz. 64(3):294-303, https://doi.org/10.1007/s00103-021-03283-3.

Mannheim24.de (2020, 25. März) Jugendliche husten Senioren an und rufen „Corona“ - nicht nur die Polizei ist entsetzt. Mannheim24.de, https://www.mannheim24.de/region/ corona-ekel-fall-radfahrer-spuckt-fussgaenger-gesicht-polizei-zeugen-ermittlungenheidelberg-13619877.html. Zugriff am 9. August 2021.

Okunew N, Theinert N (2020) Die Pandemie als Generationenkonflikt? Von „Corona-Partys“ und Moral Panics, in: Zeitgeschichte-online, https://zeitgeschichte-online.de/kommentar/diepandemie-als-generationenkonflikt. Zugriff am 9. August 2021.

Pauls P (2020, 21. März) Corona in Deutschland. Das Ende der Komfortzone (2021) Deutschlandfunk, 06:05-06:10 Uhr; Transkript zitiert nach: Okunew u. Theinert 2020, https:// www.deutschlandfunk.de/programmvorschau.281.de.html?drbm:date=21.03.2020. Zugriff am 9. August 2021.

Petter J, Eberle L (2021, 20. März) Die Wut auf Corona-Partys wächst - doch wie viele Feiern gibt es wirklich? DER SPIEGEL online/ bento.de, https://www.spiegel.de/panorama/coronapartys-wie-viele-feiern-gibt-es-wirklich-a-68fbfa4f-4fbd-403e-9900-6131a1169eae.Zugriff am 9. August 2021. 
O'Toole G (2010) Misbehaving children in ancient times. https://quoteinvestigator.com/2010/ 05/01/misbehave. Zugriff am 9. August 2021.

Rieg T (2020) Desinfektionsjournalismus. Journalistik. Zeitschrift für Journalismusforschung. Heft 2/2020, 159-171. https://journalistik.online/ausgabe-2-2020/ desinfektionsjournalismus/.

Rosenfelder A (2020, 30.4.). Der Lohn der Angst. Kommentar. Die Welt, S. 8.

RTL news (2020, 24. April) Fall Fabio (†5): Was wusste das Jugendamt? RTL news.de, https:// www.rtl.de/cms/fall-fabio-5-was-wusste-das-jugendamt-in-moenchengladbach4528678.html. Zugriff am 9. August 2021.

Salihi N (2020) Jugendbilder in Zeiten von Corona: Engagement, Verantwortung und Solidarität - jenseits von Corona-Partys. Jugendhilfeportal.de, https://www.jugendhilfeportal. de/fokus/coronavirus/artikel/jugendbilder-in-zeiten-von-corona-engagementverantwortung-und-solidaritaet-jenseits-von-corona. Zugriff am 9. August 2021.

Sartory B (2021, 15. Januar) Der Fall Fabio. WDR 5 Westblick - aktuell. (3:40 Min.) https://www1. wdr.de/nachrichten/rheinland/prozess-fabio-revision-totschlag-100.html. Zugriff am 9. August 2021.

Schnetzer S, Hurrelmann K (2021) Jugend und Corona: Wie rücksichtsvoll verhalten sich die jungen Generationen? Sonderauswertung der Studie Junge Deutsche 2021. Kempten: DATAJOCKEY.

Spittler M (2020) Corona - Eine Generationenfrage? Beitrag zur TUI Jugendstudie 2020, https:// www.tui-stiftung.de/unsere-projekte/junges-europa-die-jugendstudie-der-tui-stiftung/ corona-eine-generationenfrage. Zugriff am 9. August 2021.

Straubhaar T (2020, 11. Juni) Weil wir nicht wissen, ob Kinder „Virenschleudern“ sind, sollten Schulen öffnen. Kommentar. Welt online, https://www.welt.de/wirtschaft/ article209344491/Corona-Sind-Kinder-Virenschleudern-oder-Virenschlucker.html. Zugriff am 9. August 2021.

TUI Jugendstudie (2020, Oktober) Junges Deutschland in Zeiten von Corona. tui-stiftung.de, https://www.tui-stiftung.de/wp-content/uploads/2020/10/2020_YouGov_TUI-Stiftung_ Junges-Deutschland-in-Zeiten-von-Corona.pdf. Zugriff am 9. August 2021.

TUI Jugendstudie (2021, 16. Juni) Junges Europa 2021. So denken Menschen zwischen 16 und 26 Jahren. tui-stiftung.de, https://www.tui-stiftung.de/unsere-projekte/junges-europadie-jugendstudie-der-tui-stiftung. Zugriff am 9. August 2021.

Voigts G (2020) Vom „Jugend vergessen“ zum „Jugend ermöglichen“: Bewegungs-, Beteiligungs- und Freiräume für junge Menschen in Corona-Zeiten. In: Forum Kind Jugend Sport 1 (2), 93-99. https://doi.org/10.1007/s43594-020-00022-5.

Warkus M (2020, 24. Oktober) Die Jugend von heute. Warkus' Welt, Spektrum.de, https://www. spektrum.de/kolumne/die-jugend-von-heute/1784201. Zugriff am 9. August 2021.

Wojtuschak U (2020, 22.April) Notarzt holt Polizei: Junge (5) zu Tode misshandelt? Bild.de, Regional/ Düsseldorf, https://www.bild.de/regional/duesseldorf/duesseldorf-aktuell/ moenchengladbach-junge-5-zu-tode-misshandelt-70208808.bild.html. Zugriff am 9. August 2021.

ZDF (2020, 20. November) Generationenkonflikt durch Corona: Vertieft die Pandemie die Gräben? Aspekte (4 Min.), Beitrag in der Mediathek nicht mehr verfügbar. 